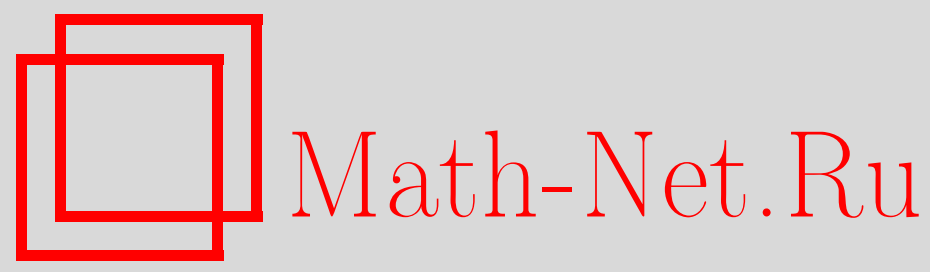

О. А. Матевосян, И. В. Филимонова, Об усреднении полулинейных параболических операторов в перфорированном цилиндре, Матем. заметки, 2005, том 78, выпуск 3, 396408

DOI: https://doi.org/10.4213/mzm2596

Использование Общероссийского математического портала Math-Net.Ru подразумевает, что вы прочитали и согласны с пользовательским соглашением http://www.mathnet.ru/rus/agreement

Параметры загрузки:

IP: 35.173 .219 .149

26 апреля 2023 г., 13:31:52

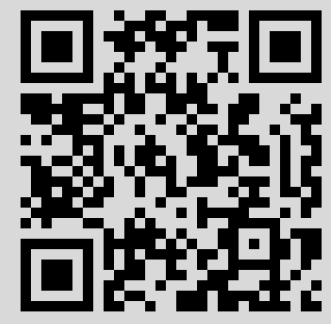




\title{
ОБ УСРЕДНЕНИИ ПОЛУЛИНЕЙНЫХ ПАРАБОЛИЧЕСКИХ ОПЕРАТОРОВ В ПЕРФОРИРОВАННОМ ЦИЛИНДРЕ
}

\author{
О. А. Матевосян, И. В. Филимонова
}

\begin{abstract}
В работе для полулинейного параболического уравнения второго порядка с младшим членом, растущим по неизвестной функции степенным образом, доказывается, что последовательность решений в перфорированном цилиндре стремится к решению в неперфорированном цилиндре, если радиусы выброшенных шаров, в параболической метрике, стремятся к нулю со скоростью, зависящей от показателя степени в младшем члене.
\end{abstract}

Библиография: 15 названий.

Изучению вопросов теории усреднения для перфорированных областей посвящено много работ (см. [1]-[3]), в которых на поведение решений при приближении к дырам ставятся какие-либо ограничения, например, - граничные условия. Однако для усреднения решений уравнений с достаточно сильньм нелинейным поглощением такие ограничения не нужны. Подобная ситуация была обнаружена и в теории устранения особенностей. В связи с этим отметим работы [4]-[7]. Вопросы усреднения полулинейных эллиптических задач в перфорированных областях были изучены в работах [8], [9] с использованием оценки Кондратьева-Ландиса $[10, \S 1$, п. 3]. Доказательства теорем настоящей работы основьваются на аналогичной оценке решения полулинейного параболического уравнения через расстояние от точки до границы области в соответствующей метрике, полученной в работе [11].

Мы будем пользоваться следующими обозначениями: $x=\left(x_{1}, \ldots, x_{n}\right) \in \mathbb{R}^{n}, n \geqslant 2$, $u=\left(u_{1}, \ldots, u_{n}\right), u_{x}=\left(u_{x_{1}}, \ldots, u_{x_{n}}\right),\left|u_{x}\right|^{2}=\sum_{i=1}^{n}\left(u_{x_{i}}\right)^{2}, u_{t}=\partial u / \partial t, 0 \leqslant t<T<\infty$; кроме того,

$\Omega \subset \mathbb{R}^{n}$-произвольное открытое подмножество $\mathbb{R}^{n}$ с границей $\partial \Omega, \bar{\Omega}=\Omega \cup \partial \Omega$ замыкание $\Omega$;

$Q=\Omega \times(0, T)$ - цилиндр в пространстве точек $(x, t) \in \mathbb{R}^{n+1}, x \in \Omega, t \in[0, T]$, $0<T<\infty$;

$S(Q)=\partial \Omega \times[0, T]-$ боковая поверхность цилиндра $Q \subset \mathbb{R}^{n+1} ; \Gamma(Q)=S(Q) \cup$ $\Upsilon_{0}(\Omega)$ - параболическая граница цилиндра $Q \subset \mathbb{R}^{n+1}$, где $\Upsilon_{t}(\Omega)=\bar{\Omega} \times\{t\}$ $\forall t \in[0, T]$

$Q\left(x_{0}, t_{0}, R, T\right)=B\left(x_{0}, R\right) \times\left[t_{0}, t_{0}+T\right]-$ цилиндр в $\mathbb{R}^{n+1}$ радиуса $R$ и высоты $T$, где $B\left(x_{0}, R\right)=\left\{x \in \mathbb{R}^{n}:\left|x-x_{0}\right| \leqslant R\right\}$ - замкнутьй шар в $\mathbb{R}^{n}$ радиуса $R$ с центром в точке $x_{0}$. 
Отметим, что цилиндр $Q\left(x_{0}, t_{0}, R, R^{2}\right)$ является шаром в параболической метрике, т.е. в метрике, в которой расстояние меж ду точками определяется следующим образом:

$$
\operatorname{dist}\left[\left(x_{1}, t_{1}\right),\left(x_{2}, t_{2}\right)\right]=\sqrt{\left(x_{1}-x_{2}\right)^{2}+\left|t_{1}-t_{2}\right|},
$$

центр которого находится в точке с координатами $\left(x_{0}, t_{0}+R / 2\right)$.

Обозначим

$\mathscr{C}(Q)$ - совокупность непрерывных в $Q$ функций;

$L_{p, r}(Q)$ - пространство, состоящее из всех измеримых на $Q$ функций с конечной нормой

$$
\left\|u ; L_{p, r}(Q)\right\|=\left(\int_{0}^{T}\left(\int_{\Omega}|u(x, t)|^{p} d x\right)^{r / p} d t\right)^{1 / r}, \quad p \geqslant 1, \quad r \geqslant 1
$$

$L_{p, r}(Q)$ будем обозначать через $L_{p}(Q)$ и норму $\left\|\cdot ; L_{p, r}(Q)\right\|$ через $\left\|\cdot ; L_{p}(Q)\right\|$ при $p=r$;

$W_{p}^{1,1}(Q)$ - пространство Соболева, состоящее из элементов $L_{p}(Q)$, имеющих обобщенные производные по $x$ и по $t$ с нормой

$$
\left\|u ; W_{p}^{1,1}(Q)\right\|=\left\|u ; L_{p}(Q)\right\|+\left\|u_{x} ; L_{p}(Q)\right\|+\left\|u_{t} ; L_{p}(Q)\right\| ;
$$

$W_{p}^{1,0}(Q)$ - пространство Соболева, состоящее из элементов $L_{p}(Q)$, имеющих обобщенные производные по $x$ с нормой

$$
\left\|u ; W_{p}^{1,0}(Q)\right\|=\left\|u ; L_{p}(Q)\right\|+\left\|u_{x} ; L_{p}(Q)\right\| .
$$

Пусть $L, \mathscr{L}$ - операторы вида

$$
L \equiv \sum_{i, j=1}^{n} \frac{\partial}{\partial x_{i}}\left(a_{i j}(x, t) \frac{\partial}{\partial x_{j}}\right), \quad \mathscr{L} \equiv L-\frac{\partial}{\partial t}
$$

где $(x, t)=\left(x_{1}, \ldots, x_{n}, t\right) \in \mathbb{R}^{n+1}$, коэффициенты $a_{i j}(x, t): \mathbb{R}^{n+1} \rightarrow \mathbb{R}^{1}$ - ограниченные измеримые функции такие, что $a_{i j}(x, t) \equiv a_{j i}(x, t)$ для всех $(x, t) \in \mathbb{R}^{n+1}$, и вьполняются условия

$$
\lambda^{-1}|\xi|^{2} \leqslant \sum_{i, j=1}^{n} a_{i j}(x, t) \xi_{i} \xi_{j} \leqslant \lambda|\xi|^{2}, \quad \lambda=\text { const }>0
$$

для всех $\xi=\left(\xi_{1}, \ldots, \xi_{n}\right) \in \mathbb{R}^{n},|\xi|^{2}=\sum_{i=1}^{n} \xi_{i}^{2}$.

Будем изучать решение задачи

$$
\begin{aligned}
\mathscr{L} u(x, t) & =f(x, t, u(x, t)), & & (x, t) \in Q=\Omega \times(0, T), \\
u(x, t) & =\varphi(x, t), & & (x, t) \in \Gamma(Q)=S(Q) \cup \Upsilon_{0}(\Omega),
\end{aligned}
$$

где $\varphi(x, t) \in W_{2}^{1,1}(Q)$, а функция $f(x, t, u): \mathbb{R}^{n+1} \times \mathbb{R}^{1} \rightarrow \mathbb{R}^{1}$ предполагается измеримой и такой, что композиция $f(x, t, u(x, t))$ является измеримой локально ограниченной функцией переменных $(x, t)$ при любой функции $u(x, t) \in W_{2}^{1,1}(Q) \cap C(Q)$. 
ОПРЕДЕЛЕНИЕ 1. Функция $u(x, t) \in W_{2,1 \text { ос }}^{1,1}(Q) \cap C(Q)$ называется обобщенным $р е$ шением задачи $(2),(3)$, если для любой функции $\psi(x, t) \in W_{2}^{1,0}(Q)$ она удовлетворяет интегральному соотношению

$$
\sum_{i, j=1}^{n} \int_{Q} a_{i j}(x, t) u_{x_{j}} \psi_{x_{i}} d x d t+\int_{Q} u_{t}(x, t) \psi(x, t) d x d t+\int_{Q} f(x, t, u) \psi(x, t) d x d t=0
$$

и при любой функции $\chi(x, t) \in C^{\infty}(Q)$, равной нулю в окрестности $\Upsilon_{T}(\Omega)$, вьполнено включение $\chi(x, t)(u(x, t)-\varphi(x, t)) \in \stackrel{\circ}{W}_{2}^{1,1}(Q)$.

Вопросы разрешимости, а также единственности решения задачи $(2),(3)$, изучены в $[12$, гл. V, $\S 6]$.

1. Пусть в области $Q$ лежат $m$ цилиндров вида $Q\left(y_{k, \varepsilon}, t_{k, \varepsilon}, R_{\varepsilon}, T_{\varepsilon}\right)$ с центрами в точках $y_{k, \varepsilon}$ одинакового радиуса $R_{\varepsilon}$ и высотами $T_{\varepsilon}, \varepsilon=m^{-1}$ - малый параметр, $m$ - натуральное число; $k=1, \ldots, m$. Отметим, что $R_{\varepsilon}$ и $T_{\varepsilon}$ связаны соотношением $T_{\varepsilon}=\left(R_{\varepsilon}\right)^{2}$, т.е. $Q\left(y_{k, \varepsilon}, t_{k, \varepsilon}, R_{\varepsilon}, R_{\varepsilon}^{2}\right)$ - шары в параболической метрике радиусов $R_{\varepsilon}$ с центрами в точках $\left(y_{k, \varepsilon}, t_{k, \varepsilon}+R_{\varepsilon} / 2\right)$. Будем так же предполагать, что расстояние между центрами этих шаров не меньше, чем $4 R_{\varepsilon}$.

Положим $Q_{\varepsilon}=Q \backslash Q\left(R_{\varepsilon}, T_{\varepsilon}\right)$, где $Q\left(R_{\varepsilon}, T_{\varepsilon}\right)=\bigcup_{k=1}^{m} Q\left(y_{k, \varepsilon}, t_{k, \varepsilon}, R_{\varepsilon}, T_{\varepsilon}\right)$.

Пусть $u_{\varepsilon}(x, t)$ - решение задачи

$$
\begin{aligned}
& \mathscr{L} u_{\varepsilon}(x, t)=a(x, t)\left|u_{\varepsilon}(x, t)\right|^{\sigma-1} u_{\varepsilon}(x, t), \quad(x, t) \in Q_{\varepsilon}, \\
& u_{\varepsilon}(x, t)=\varphi(x, t) \text {, } \\
& (x, t) \in S(Q) \text {, } \\
& u_{\varepsilon}(x, 0)=u_{0}(x) \text {, } \\
& x \in \Omega \text {, }
\end{aligned}
$$

где $a(x, t)$ - ограниченная измеримая функция, $a(x, t) \geqslant a_{0}=$ const $>0, \varphi(x) \in W_{2}^{1,1}\left(Q_{\varepsilon}\right)$.

ОПРЕДЕЛЕНИЕ 2. Функция $u_{\varepsilon}(x, t) \in W_{2,1 \text { ос }}^{1,1}\left(Q_{\varepsilon}\right) \cap C\left(Q_{\varepsilon}\right)$ назьвается обобщенны.м решением задачи (5)-(7), если для любой функции $\psi(x, t) \in W_{2}^{1,0}\left(Q_{\varepsilon}\right)$ она удовлетворяет интегральному соотношению

$$
\begin{gathered}
\sum_{i, j=1}^{n} \int_{Q_{\varepsilon}} a_{i j}(x, t) u_{x_{j}} \psi_{x_{i}} d x d t+\int_{Q_{\varepsilon}} u_{t}(x, t) \psi(x, t) d x d t \\
\quad+\int_{Q_{\varepsilon}} a(x, t)\left|u_{\varepsilon}(x, t)\right|^{\sigma-1} u_{\varepsilon}(x, t) \psi(x, t) d x d t=0,
\end{gathered}
$$

принимает значение $u_{0}(x)$ при $t=0$ и при любой функции $\chi(x, t) \in C^{\infty}(Q)$, равной нулю в окрестностях $Q\left(R_{\varepsilon}, T_{\varepsilon}\right)$ и $\Upsilon_{T}(\Omega)$, выполнено включение $\chi(x, t)\left(u_{\varepsilon}(x, t)-\varphi(x, t)\right) \in$ $W_{2}^{1,1}\left(Q_{\varepsilon}\right)$.

Для задачи (5)-(7) не выполняются, вообще говоря, теоремы единственности, так как на границах дырок перфорированной области $Q_{\varepsilon}$ не заданы никакие граничные условия. Мы покажем, что при некоторых условиях предел решений задачи (5)-(7) будет существовать, причем этот предел не зависит от выбора последовательности $u_{\varepsilon}(x, t)$; здесь $u_{\varepsilon}(x, t)$ - любое решение задачи $(5)-(7)$. 
Пусть $u(x, t)$ - решение следующей задачи:

$$
\begin{aligned}
\mathscr{L} u(x, t) & =a(x, t)|u(x, t)|^{\sigma-1} u(x, t), & (x, t) & \in Q, \\
u(x, t) & =\varphi(x, t), & (x, t) & \in S(Q), \\
u(x, 0) & =u_{0}(x), & x & \in \Omega .
\end{aligned}
$$

TEOPEMA 1. Пусть $\sigma>(n+2) / n u$

$$
R_{\varepsilon}=\varepsilon^{\alpha}, \quad T_{\varepsilon}=\varepsilon^{2 \alpha}, \quad \text { əде } \quad \alpha>\frac{\sigma-1}{(\sigma-1) n-2} .
$$

Тогда решение задачи (5)-(7) стремится $к$ решению задачи (8)-(10) в следующем смысле: для любого положительного постоянного $d$

$$
\sup _{(x, t) \in Q_{\varepsilon, d}}\left|u_{\varepsilon}(x, t)-u(x, t)\right| \rightarrow 0 \quad n p u \quad \varepsilon \rightarrow 0
$$

$2 \partial e$

$$
Q_{\varepsilon, d}=\left\{(x, t) \in Q_{\varepsilon}: \operatorname{dist}\left[(x, t), \partial Q\left(R_{\varepsilon}, T_{\varepsilon}\right)\right]>2 d u\left|t-t_{k, \varepsilon}\right|>d ; k=1, \ldots, m\right\} .
$$

Отметим, что при этом общий объем дырок $\operatorname{Vol}\left[Q\left(R_{\varepsilon}, T_{\varepsilon}\right)\right]$ стремится к нулю при $\varepsilon \rightarrow 0$.

СлЕДСТВИЕ. Если в перфорированной области $Q_{\varepsilon}$ существует множество, отделенное от всех дырок фиксированной постоянной, то на этом множсестве сходимость будет равномерной.

Лемма 1. Для любых $a, b \in \mathbb{R} u \sigma>1$ имеет место неравенство

$$
\left.|| a\right|^{\sigma-1} a-|b|^{\sigma-1} b\left|\geqslant C_{\sigma}\right| a-\left.b\right|^{\sigma}=C_{\sigma}|a-b|^{\sigma-1}|a-b|,
$$

әде $C_{\sigma}$-некоторая положительная постоянная, зависящая от $\sigma$, но не зависящая om $a u b$.

ДоКАЗАТЕЛЬСТВО ТЕОРЕМЫ 1. Для разности $w_{\varepsilon}(x, t)=u_{\varepsilon}(x, t)-u(x, t)$ имеем

$$
\mathscr{L} w_{\varepsilon}(x, t)-a(x, t)\left(\left|u_{\varepsilon}(x, t)\right|^{\sigma-1} u_{\varepsilon}(x, t)-|u(x, t)|^{\sigma-1} u(x, t)\right)=0 .
$$

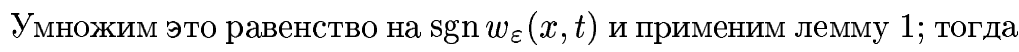

$$
\begin{aligned}
0 & =\operatorname{sgn} w_{\varepsilon}(x, t) \mathscr{L} w_{\varepsilon}(x, t)-\left.a(x, t)|| u_{\varepsilon}(x, t)\right|^{\sigma-1} u_{\varepsilon}(x, t)-|u(x, t)|^{\sigma-1} u(x, t) \mid \\
& \leqslant \operatorname{sgn} w_{\varepsilon}(x, t) \mathscr{L} w_{\varepsilon}(x, t)-C_{\sigma} a_{0}\left|u_{\varepsilon}(x, t)-u(x, t)\right|^{\sigma} \\
& =\operatorname{sgn} w_{\varepsilon}(x, t) \mathscr{L} w_{\varepsilon}(x, t)-C_{\sigma} a_{0}\left|w_{\varepsilon}(x, t)\right|^{\sigma}, \quad(x, t) \in Q_{\varepsilon} .
\end{aligned}
$$

Таким образом, функция $w_{\varepsilon}(x, t)$ удовлетворяет неравенству

$$
\operatorname{sgn} w_{\varepsilon}(x, t) \mathscr{L} w_{\varepsilon}(x, t)-b_{0}\left|w_{\varepsilon}(x, t)\right|^{\sigma} \geqslant 0, \quad(x, t) \in Q_{\varepsilon},
$$


с граничньм условием

$$
w_{\varepsilon}(x, t)=0, \quad(x, t) \in S(Q)=\partial \Omega \times[0, T],
$$

и с нулевым начальным условием

$$
w_{\varepsilon}(x, 0)=0, \quad x \in \Omega
$$

здесь $b_{0}=C_{\sigma} a_{0}$.

Согласно теореме 8.2 работы [11] для решений полулинейных параболических уравнений вида (8) имеет место оценка

$$
\left|w_{\varepsilon}(x, t)\right| \leqslant C\left(\min \left(R^{2}, T\right)\right)^{1 /(1-\sigma)},
$$

где $R, T$ - соответственно радиус и высота цилиндра, в котором определено решение $u(x, t)$ уравнения (8). Аналогично работе [10] эту оценку можно получить и для решений неравенств вида (13). Буквой $C$, здесь и далее, будем обозначать любую положительную постоянную, не зависящую от $x, t$ и $\varepsilon$.

Предположим, что $u_{\varepsilon}(x, t)$ не стремится к $u(x, t)$ в смысле $(12)$ при $\varepsilon \rightarrow 0$. Тогда найдется положительная постоянная $C_{0}$ такая, что для любого $\varepsilon_{0}=m_{0}^{-1}$ существуют $\varepsilon=m^{-1}<\varepsilon_{0}$ и $\left(x_{\varepsilon}, t_{\varepsilon}\right) \in Q_{\varepsilon, d}$ такие, что $\left|w_{\varepsilon}\left(x_{\varepsilon}, t_{\varepsilon}\right)\right|>C_{0}$, т.е. $w_{\varepsilon}\left(x_{\varepsilon}, t_{\varepsilon}\right)>C_{0}$ либо $w_{\varepsilon}\left(x_{\varepsilon}, t_{\varepsilon}\right)<-C_{0}$. Будем считать, что первый случай реализуется при различных $\varepsilon$ бесконечно много раз (для второго случая $w_{\varepsilon}\left(x_{\varepsilon}, t_{\varepsilon}\right)<-C_{0}$ рассмотрение аналогично).

Обозначим через $\Gamma\left(x, t, x_{0}, t_{0}\right)$ фундаментальное решение оператора $\mathscr{L}$, имеюшее особенность в точке $\left(x_{0}, t_{0}\right)$, т.е. $\mathscr{L} \Gamma(x, t)=\delta\left(x_{0}, t_{0}\right)$. Известно [13] (см. также [14]), что такое фундаментальное решение сушествует и удовлетворяет неравенствам

$$
C_{1} \frac{\exp \left(-C_{3}\left|x-x_{0}\right|^{2} /\left(t-t_{0}\right)\right)}{\left(t-t_{0}\right)^{n / 2}} \leqslant \Gamma\left(x, t, x_{0}, t_{0}\right) \leqslant C_{1} \frac{\exp \left(-C_{4}\left|x-x_{0}\right|^{2} /\left(t-t_{0}\right)\right)}{\left(t-t_{0}\right)^{n / 2}}
$$

где положительные постоянные $C_{1}$ и $C_{2}$ зависят от $\lambda$ из условия (1).

Положим

$$
g_{\varepsilon}(x, t)=\frac{1}{m} \sum_{k=1}^{m} \Gamma\left(x, t, y_{k, \varepsilon}, t_{k, \varepsilon}-T_{\varepsilon}\right)
$$

Рассмотрим функцию

$$
v_{\varepsilon}(x, t)=w_{\varepsilon}(x, t)-h_{\varepsilon}(x, t),
$$

где $h_{\varepsilon}(x, t)=\left(C_{0} / 2\right)\left(g_{\varepsilon}(x, t) / g_{\varepsilon}\left(x_{\varepsilon}, t_{\varepsilon}\right)\right)$. Тогда выполняется $v_{\varepsilon}\left(x_{\varepsilon}, t_{\varepsilon}\right)=C_{0} / 2>0$.

Покажем, используя принцип максимума, что это невозможно.

Используя предположение, что параболическое расстояние между точками $\left(y_{k, \varepsilon}, t_{k, \varepsilon}\right)$ при малых $\varepsilon$ больше, чем $4 R_{\varepsilon}$, согласно (16) на границах цилиндров $Q\left(y_{k, \varepsilon}, t_{k, \varepsilon}, 2 R_{\varepsilon}\right.$, $\left.4 T_{\varepsilon}\right)$, коаксиальных с $Q\left(y_{k, \varepsilon}, t_{k, \varepsilon}, R_{\varepsilon}, T_{\varepsilon}\right)$, вьполнено неравенство

$$
\left|w_{\varepsilon}(x, t)\right|<C R_{\varepsilon}^{2 /(1-\sigma)} .
$$


Из нижней оценки (17) заключаем, что на границах цилиндров $Q\left(y_{k, \varepsilon}, t_{k, \varepsilon}, 2 R_{\varepsilon}, 4 T_{\varepsilon}\right)$ имеют место неравенства

$$
g_{\varepsilon}(x, t)>\varepsilon C_{1} \frac{\exp \left(-4 C_{3}\left|R_{\varepsilon}\right|^{2} /\left(t-t_{k, \varepsilon}+T_{\varepsilon}\right)\right)}{\left(t-t_{k, \varepsilon}+T_{\varepsilon}\right)^{n / 2}}>\varepsilon C_{1} \frac{\exp \left(-8 C_{3}\left|R_{\varepsilon}\right|^{2} / T_{\varepsilon}\right)}{\left(5 T_{\varepsilon}\right)^{n / 2}} .
$$

Потребуем вьполнения соотношения

$$
R_{\varepsilon}^{2 /(1-\sigma)}=\mathbf{o}\left(\varepsilon C_{1} \frac{\exp \left(-8 C_{3}\left|R_{\varepsilon}\right|^{2} / T_{\varepsilon}\right)}{\left(T_{\varepsilon} / 2\right)^{n / 2}}\right) \quad \text { при } \varepsilon \rightarrow 0 .
$$

Так как показатель экспоненты в силу условия теоремы ограничен, это соотношение равносильно $R_{\varepsilon}^{2 /(1-\sigma)}=\mathbf{o}\left(\varepsilon T_{\varepsilon}^{-n / 2}\right)$ при $\varepsilon \rightarrow 0$, т.е. когда $1-\alpha n<2 \alpha /(1-\sigma)$. Таким образом, получаем, что при $\sigma>(n+2) / n$ для выполнения $(22)$ необходимо, чтобы $\alpha>(\sigma-1) /((\sigma-1) n-2)$.

Так как точка $\left(x_{\varepsilon}, t_{\varepsilon}\right) \in Q_{\varepsilon, d}$, то $g_{\varepsilon}\left(x_{\varepsilon}, t_{\varepsilon}\right)<c / d^{n / 2}$. Поэтому в силу $(22)$ на границах цилиндров $Q\left(y_{k, \varepsilon}, t_{k, \varepsilon}, 2 R_{\varepsilon}, 4 T_{\varepsilon}\right)$ будет выполнено неравенство $w_{\varepsilon}(x, t)<g_{\varepsilon}(x, t)$, т.е. выполнено $v_{\varepsilon}(x, t)<0$.

Для того, чтобы исключить из области точки особенностей фундаментальных решений, дополним цилиндры радиусов $2 R_{\varepsilon}$ внутренностями параболоидов

$$
t-t_{k, \varepsilon}+T_{\varepsilon}=\frac{1}{4}\left(\left|x-y_{k, \varepsilon}\right|\right)^{2} .
$$

На границах параболоидов показатель экспоненты постоянен и, следовательно,

$$
g(x, t)>\varepsilon \frac{k}{\left(t-t_{k, \varepsilon}+T_{\varepsilon}\right)^{n / 2}}>\varepsilon \frac{4 k}{\left(R_{\varepsilon}\right)^{n}}=\varepsilon \frac{4 k}{\left(T_{\varepsilon}\right)^{n / 2}} .
$$

Поэтому на границах параболоидов также вьполнено $v_{\varepsilon}(x, t)<0$.

При $(x, t) \in \partial \Omega \times[0, T]$ согласно $(14)$ и (18) имеем $w_{\varepsilon}(x, t)=0, g_{\varepsilon}(x, t) \geqslant 0$ и поэтому $v_{\varepsilon}(x, t) \leqslant 0$ при $(x, t) \in S(Q)$. При $t=0: w_{\varepsilon}(x, t)=g_{\varepsilon}(x, t)=0$, имеем $v_{\varepsilon}(x, t) \leqslant 0$.

Кроме того, из оценок (16), (17) с учетом определения (18) следует, что $w_{\varepsilon}(x, t) \rightarrow 0$ и $g_{\varepsilon}(x, t) \rightarrow 0$ при $|x| \rightarrow \infty$. Таким образом, из принципа максимума заключаем, что $v_{\varepsilon}(x, t) \leqslant 0$ на параболической границе $\Gamma(Q)$.

Определим множество $D \equiv\left\{(x, t) \in Q_{\varepsilon}: v_{\varepsilon}(x, t)>0\right\} \neq \varnothing$. Обозначим $D_{0}$ компоненту связности множества $D$, содержащую точку $\left(x_{\varepsilon}, t_{\varepsilon}\right)$.

Покажем, что $\mathscr{L} v_{\varepsilon}(x, t)>0$ при $(x, t) \in D$. Действительно, по определению множества $D$

$$
u_{\varepsilon}(x, t)-u(x, t)=w_{\varepsilon}(x, t)>h_{\varepsilon}(x, t)>0,
$$

т.е. $u_{\varepsilon}(x, t)>u(x, t)$ при $(x, t) \in D$. Тогда

$$
\begin{aligned}
\mathscr{L} v_{\varepsilon}(x, t) & =\mathscr{L}\left(u_{\varepsilon}(x, t)-u(x, t)\right)-\mathscr{L} h_{\varepsilon}(x, t) \\
& =a(x, t)\left[\left|u_{\varepsilon}(x, t)\right|^{\sigma-1} u_{\varepsilon}(x, t)-|u(x, t)|^{\sigma-1} u(x, t)\right]>0
\end{aligned}
$$

Поскольку $v_{\varepsilon}\left(x_{\varepsilon}, t_{\varepsilon}\right)>0$ согласно $(19), v_{\varepsilon}(x, t)<0$ при $x \in \partial\left(Q\left(R_{\varepsilon}, T_{\varepsilon}\right)\right), v_{\varepsilon}(x, 0)<0$ и $v_{\varepsilon}(x, t) \rightarrow 0$ при $|x| \rightarrow \infty$, то функция $v_{\varepsilon}(x, t)$ достигает локального максимума во 
внутренней точке области $D_{0}$, что с учетом (23) противоречит принципу максимума для функции $v_{\varepsilon}(x, t)$.

Следовательно, наше предположение неверно, т.е. $u_{\varepsilon}(x, t)$ стремится к $u(x, t)$ в смысле (12) при $\varepsilon \rightarrow 0$. Теорема 1 доказана.

Для объема шара радиуса $R$ в пространстве $\mathbb{R}^{n}$ вьполнено равенство

$$
\operatorname{Vol}[B(0, R)]=\mu_{n} R^{n}
$$

где постоянная $\mu_{n}$ - объем единичного шара в $\mathbb{R}^{n}-$ зависит только от $n$. Оценим общий объем дырок:

$$
\begin{aligned}
\left|\bigcup_{k=1}^{m} Q\left(y_{k, \varepsilon}, t_{k, \varepsilon}, R_{\varepsilon}, T\right)\right| & \leqslant m \operatorname{Vol}\left[Q\left(0,0, R_{\varepsilon}, T\right)\right] \\
& =T_{\varepsilon} \mu_{n} m R_{\varepsilon}^{n} \sim \varepsilon^{-1+(2+n) \alpha} \rightarrow 0 \quad \text { при } \varepsilon \rightarrow 0,
\end{aligned}
$$

если только $\alpha>1 /(n+2)$.

Отметим, что не сложно модернизировать доказательство теоремы 1 для случая, когда $R_{\varepsilon} \sim \varepsilon^{\alpha}$ и $T_{\varepsilon} \sim \varepsilon^{2 \alpha}$.

Пусть коэффициенты оператора не зависят от $t$ и $n>2$. Из оценки (16), так как $w(x, t)$ обращается в нуль при $t=0$, можно исключить зависимость от $T$. Действительно, $w(x, t)$ всегда можно считать решением в достаточно высоком цилиндре фиксированного радиуса, если доопределить $w(x, t)$ нулем при $t<0$. Рассматривая фундаментальные решения эллиптического оператора, можно доказать следующую теорему для перфорированных областей, из которых вместо шаров выкидьваются цилиндры.

TEOPEMA $1^{\prime}$. Пyсmb $n>2, \sigma>n /(n-2) u$

$$
R_{\varepsilon} \sim \varepsilon^{\alpha} \quad \text { npu } \quad \varepsilon \rightarrow 0, \quad \text { əде } \quad \alpha>\frac{\sigma-1}{(n-2) \sigma-n} .
$$

Тогда решение задачи (5)-(7) стремится $к$ решению задачи (8)-(10) в следующем смысле: для любого положительного постоянного $d$

$$
\sup _{x \in \Omega_{\varepsilon, d}, t \in[0, T]}\left|u_{\varepsilon}(x, t)-u(x, t)\right| \rightarrow 0 \quad \text { npu } \quad \varepsilon \rightarrow 0
$$

$2 \partial e$

$$
\Omega_{\varepsilon, d}=\left\{x \in \Omega: \operatorname{dist}\left(x, \partial B\left(y_{k, \varepsilon}, R_{\varepsilon}\right)\right)>d ; k=1, \ldots, m\right\} .
$$

Доказательство проводится так же, как в работах [8], [9] для эллиптических операторов.

2. Пусть теперь в области $Q$ лежат $m$ цилиндров вида $Q\left(y_{k, \varepsilon}, 0, R_{\varepsilon}, T\right)$ с центрами в точках $y_{k, \varepsilon}$ одинакового радиуса $R_{\varepsilon}$ и высотами $T, \varepsilon=m^{-1}$ - малый параметр, $m$ натуральное число; $k=1, \ldots, m$. Будем предполагать, что расстояние между центрами этих шаров не менее, чем $8 R_{\varepsilon}$.

Пусть $Q_{\varepsilon}=Q \backslash Q\left(R_{\varepsilon}, T\right), Q\left(R_{\varepsilon}, T\right)=\bigcup_{k=1}^{m} Q\left(y_{k, \varepsilon}, 0, R_{\varepsilon}, T\right)$. 
ЛЕмма 2 (обобщенное неравенство Юнга). Пусть $a, b, \beta>0,0<\alpha<1$. Тогда

$$
a b \leqslant \frac{\alpha}{\beta} a^{1 / \alpha}+\frac{1-\alpha}{\beta-\alpha /(1-\alpha)} b^{1 /(1-\alpha)} .
$$

В частности, при $\alpha=1 / 2$ имеем $2 a b \leqslant a^{2} / \beta+\beta b^{2}$.

Положим $Q\left(4 R_{\varepsilon}, T\right)=\bigcup_{k=1}^{m} Q\left(y_{k, \varepsilon}, 0,4 R_{\varepsilon}, T\right)$.

Теорема 2. При любом $\sigma>1 u R_{\varepsilon}=O\left(\varepsilon^{\gamma}\right) n p u \varepsilon \rightarrow 0$, әде $\gamma>1 /(n-2)$, выполнено

$$
\int_{Q \backslash Q\left(4 R_{\varepsilon}, T\right)} \frac{\left|\nabla\left(u_{\varepsilon}(x, t)-u(x, t)\right)\right|^{2}+\left|u_{\varepsilon}(x, t)-u(x, t)\right|^{\sigma+1}}{1+\left|u_{\varepsilon}(x, t)-u(x, t)\right|^{2}} d x d t \rightarrow 0 \quad \text { npu } \quad \varepsilon \rightarrow 0 .
$$

ДокАЗАТЕЛЬСтво. Рассмотрим функцию $\theta_{\varepsilon}(x) \in C^{\infty}(\Omega)$ такую, что $0 \leqslant \theta_{\varepsilon}(x) \leqslant 1$, $x \in \Omega$,

$$
\theta_{\varepsilon}(x)=\left\{\begin{array}{l}
1, x \in \Omega \backslash \bigcup_{k=1}^{m} B\left(y_{k, \varepsilon}, 4 R_{\varepsilon}\right), \\
0, x \in \bigcup_{k=1}^{m} B\left(y_{k, \varepsilon}, 2 R_{\varepsilon}\right)
\end{array}\right.
$$

$\left|\nabla \theta_{\varepsilon}(x)\right|<C_{\Omega} / R_{\varepsilon}, x \in \Omega ;$ здесь $C_{\Omega}$ зависит от области $\Omega$ и не зависит от $\varepsilon$.

Рассмотрим функцию

$$
\psi(x, t)=\theta_{\varepsilon}^{p}(x) \eta\left(u_{\varepsilon}(x, t)-u(x, t)\right)
$$

где

$$
\eta(\tau)= \begin{cases}\tau, & |\tau| \leqslant 1 \\ |\tau|^{q-1} \tau, & |\tau|>1\end{cases}
$$

$q>-1$ такое, что $\sigma>(n+2 q) /(n-2)$ (отметим, что такое $q$ можно подобрать при любом $\sigma>1) ; p=p(q)>2$ - решение уравнения $p=(p-2)(\sigma+q) /(1+q)$, т.е.

$$
p=2 \frac{\sigma+q}{\sigma-1}
$$

требуемое неравенство $p>2$ вьполнено при требуемом условии на $q>-1$.

Отметим также, что функцию $\psi(x, t)$ можно подставлять в интегральное тождество из определения обобщенного решения, так как она принадлежит классу $W_{2}^{1,0}\left(Q_{\varepsilon}\right)$ в силу правила цепного дифференцирования [15] и обращается в нуль на границе $\partial Q_{\varepsilon}$, на границе $\partial Q$ за счет того, что разница $u_{\varepsilon}(x, t)-u(x, t)$ обращается в нуль на $\partial Q$, а на гранище дырок за счет множителя $\theta_{\varepsilon}^{p}(x)$. Отметим также, что поскольку на всех дырках этот множитель равен нулю, в интегральном тождестве мы можем в качестве области интегрирования писать $Q$.

Как и в доказательстве теоремы 1 , обозначим $w_{\varepsilon}(x, t)=u_{\varepsilon}(x, t)-u(x, t)$. Для уравнения

$$
L w_{\varepsilon}(x, t)-\frac{\partial w_{\varepsilon}(x, t)}{\partial t}=a(x, t)\left(\left|u_{\varepsilon}(x, t)\right|^{\sigma-1} u_{\varepsilon}(x, t)-|u(x, t)|^{\sigma-1} u(x, t)\right)
$$


запишем интегральное тождество, взяв в качестве пробной функции $\psi(x, t)$ из $(24)$; получим

$$
\begin{aligned}
& \int_{Q}\left(\sum_{i, j=1}^{n} \frac{\partial}{\partial x_{i}}\left(a_{i j}(x, t) \frac{\partial w_{\varepsilon}(x, t)}{\partial x_{j}}\right)-\frac{\partial w_{\varepsilon}(x, t)}{\partial t}\right) \psi(x, t) d x d t \\
& \quad=\int_{Q} a(x, t)\left(\left|u_{\varepsilon}(x, t)\right|^{\sigma-1} u_{\varepsilon}(x, t)-|u(x, t)|^{\sigma-1} u(x, t)\right) \psi(x, t) d x d t
\end{aligned}
$$

Обозначим

$$
Q_{1}=\left\{(x, t) \in Q:\left|u_{\varepsilon}(x, t)-u(x, t)\right| \leqslant 1\right\} \subset Q
$$

и

$$
Q_{2}=\left\{(x, t) \in Q:\left|u_{\varepsilon}(x, t)-u(x, t)\right|>1\right\} \subset Q .
$$

Оценим правую часть равенства (26), используя лемму 1 при $a=u_{\varepsilon}(x, t), b=u(x, t)$ :

$$
\begin{aligned}
& \int_{Q} a(x, t)\left(\left|u_{\varepsilon}(x, t)\right|^{\sigma-1} u_{\varepsilon}(x, t)-|u(x, t)|^{\sigma-1} u(x, t)\right) \psi(x, t) d x d t \\
& =\int_{Q_{1}} a(x, t)\left(\left|u_{\varepsilon}(x, t)\right|^{\sigma-1} u_{\varepsilon}(x, t)-|u(x, t)|^{\sigma-1} u(x, t)\right) w_{\varepsilon}(x, t) \theta_{\varepsilon}^{p}(x) d x d t \\
& \quad+\int_{Q_{2}} a(x, t)\left(\left|u_{\varepsilon}(x, t)\right|^{\sigma-1} u_{\varepsilon}(x, t)-u(x, t)^{\sigma-1} u(x, t)\right)\left|w_{\varepsilon}(x, t)\right|^{q-1} w_{\varepsilon}(x, t) \theta_{\varepsilon}^{p}(x) d x d t \\
& \geqslant a_{0} C_{\sigma} \int_{Q_{1}}\left|w_{\varepsilon}(x, t)\right|^{\sigma+1} \theta_{\varepsilon}^{p}(x) d x d t+a_{0} C_{\sigma} \int_{Q_{2}}\left|w_{\varepsilon}(x, t)\right|^{\sigma+q} \theta_{\varepsilon}^{p}(x) d x d t .
\end{aligned}
$$

После тождественных преобразований и интегрирования по частям в левой части равенства (26) получим

$$
\begin{aligned}
J_{1}+ & J_{2}+J_{3}+J_{4} \\
\equiv & -\int_{Q_{1}} \sum_{i, j=1}^{n} a_{i j}(x, t) \frac{\partial w_{\varepsilon}(x, t)}{\partial x_{j}} \frac{\partial w_{\varepsilon}(x, t)}{\partial x_{i}} \theta_{\varepsilon}^{p}(x) d x d t \\
& -\int_{Q_{1}} \sum_{i, j=1}^{n} a_{i j}(x, t) \frac{\partial w_{\varepsilon}(x, t)}{\partial x_{j}} w_{\varepsilon}(x, t) p \theta_{\varepsilon}^{p-1}(x) \frac{\partial \theta_{\varepsilon}(x)}{\partial x_{i}} d x d t \\
& -\int_{Q_{2}} \sum_{i, j=1}^{n} a_{i j}(x, t) \frac{\partial w_{\varepsilon}(x, t)}{\partial x_{j}} q\left|w_{\varepsilon}(x, t)\right|^{q-1} \frac{\partial w_{\varepsilon}(x, t)}{\partial x_{i}} \theta_{\varepsilon}^{p}(x) d x d t \\
& -\int_{Q_{2}} \sum_{i, j=1}^{n} a_{i j}(x, t) \frac{\partial w_{\varepsilon}(x, t)}{\partial x_{j}}\left|w_{\varepsilon}(x, t)\right|^{q-1} w_{\varepsilon}(x, t) p \theta_{\varepsilon}^{p-1}(x) \frac{\partial \theta_{\varepsilon}(x)}{\partial x_{i}} d x d t .
\end{aligned}
$$

Оценим первое и третье слагаемые правой части тождества (28), учитьвая (1):

$$
\begin{aligned}
& J_{1} \leqslant-\frac{1}{\lambda} \int_{Q_{1}}\left|\nabla w_{\varepsilon}(x, t)\right|^{2} \theta_{\varepsilon}^{p}(x) d x d t \\
& J_{3} \leqslant-\frac{q}{\lambda} \int_{Q_{2}}\left|\nabla w_{\varepsilon}(x, t)\right|^{2}\left|w_{\varepsilon}(x, t)\right|^{q-1} \theta_{\varepsilon}^{p}(x) d x .
\end{aligned}
$$


Для оценки второго и четвертого слагаемых правой части тождества $(28)$ используется неравенство Юнга при $\alpha=1 / 2, a=\left|\nabla w_{\varepsilon}(x, t)\right|, b=\left|\nabla \theta_{\varepsilon}(x)\right|$. Выбирая $\beta=p \lambda^{2} / \theta_{\varepsilon}(x)$ и $\beta=p \lambda^{2}\left|w_{\varepsilon}(x, t)\right| /\left(q \theta_{\varepsilon}(x)\right)$ при каждом $(x, t)$, соответственно получаем

$$
\begin{aligned}
& J_{2} \leqslant p \lambda \int_{Q_{1}}\left|\nabla w_{\varepsilon}(x, t)\right|\left|\nabla \theta_{\varepsilon}(x)\right| \theta_{\varepsilon}^{p-1}(x) d x d t \\
& \leqslant p \lambda \int_{Q_{1}} \theta_{\varepsilon}^{p-1}(x)\left(\frac{\theta_{\varepsilon}(x)}{2 p \lambda^{2}}\left|\nabla w_{\varepsilon}(x, t)\right|^{2}+\frac{p \lambda^{2}}{2 \theta_{\varepsilon}(x)}\left|\nabla \theta_{\varepsilon}(x)\right|^{2}\right) d x d t \\
& =\frac{1}{2 \lambda} \int_{Q_{1}}\left|\nabla w_{\varepsilon}(x, t)\right|^{2} \theta_{\varepsilon}^{p}(x) d x d t+C_{4} \int_{Q_{1}}\left|\nabla \theta_{\varepsilon}(x)\right|^{2} \theta_{\varepsilon}^{p-2}(x) d x d t, \\
& J_{4} \leqslant p \lambda \int_{Q_{2}}\left|\nabla w_{\varepsilon}(x, t)\right|\left|\nabla \theta_{\varepsilon}(x)\right|\left|w_{\varepsilon}(x, t)\right|^{q} \theta_{\varepsilon}^{p-1}(x) d x d t \\
& \leqslant p \lambda \int_{Q_{2}} \theta_{\varepsilon}^{p-1}(x)\left|w_{\varepsilon}(x, t)\right|^{q} \\
& \times\left(\frac{q \theta_{\varepsilon}(x)}{2 p \lambda^{2}\left|w_{\varepsilon}(x, t)\right|}\left|\nabla w_{\varepsilon}(x, t)\right|^{2}+\frac{p \lambda^{2}\left|w_{\varepsilon}(x, t)\right|}{2 q \theta_{\varepsilon}(x)}\left|\nabla \theta_{\varepsilon}(x)\right|^{2}\right) d x d t \\
& =\frac{q}{2 \lambda} \int_{Q_{2}}\left|\nabla w_{\varepsilon}(x, t)\right|^{2}\left|w_{\varepsilon}(x, t)\right|^{q-1} \theta_{\varepsilon}^{p}(x) d x d t \\
& +C_{5} \int_{Q_{2}}\left|\nabla \theta_{\varepsilon}(x)\right|^{2}\left|w_{\varepsilon}(x, t)\right|^{q+1} \theta_{\varepsilon}^{p-2}(x) d x d t,
\end{aligned}
$$

где $C_{4}=p^{2} \lambda^{3} / 2, C_{5}=p^{2} \lambda^{3} /(2 q)$.

Теперь оценим второе слагаемое правой части неравенства $(32)$, полагая в неравенстве Юнга $a=\left|w_{\varepsilon}(x, t)\right|^{q+1} \theta_{\varepsilon}^{p-2}(x), b=\left|\nabla \theta_{\varepsilon}(x)\right|^{2}$ при $\alpha=(1+q) /(\sigma+q)$ и выбрав $\beta=2 C_{5} \alpha /\left(a_{0} C_{\sigma}\right)$; с учетом $(25)$ получаем

$$
\begin{aligned}
& C_{5} \int_{Q_{2}}\left|\nabla \theta_{\varepsilon}(x)\right|^{2}\left|w_{\varepsilon}(x, t)\right|^{q+1} \theta_{\varepsilon}^{p-2}(x) d x d t \\
& \quad \leqslant \frac{a_{0} C_{\sigma}}{2} \int_{Q_{2}}\left|w_{\varepsilon}(x, t)\right|^{\sigma+q} \theta_{\varepsilon}^{p}(x) d x d t+C_{6} \int_{Q_{2}}\left|\nabla \theta_{\varepsilon}(x)\right|^{p} d x d t
\end{aligned}
$$

где

$$
\begin{aligned}
C_{6} & =C_{5} \frac{\sigma-1}{\sigma+q}\left(\frac{2 C_{5}}{a_{0} C_{\sigma}} \frac{1+q}{\sigma+q}\right)^{(q+1) /(\sigma-1)} \\
& =\frac{\sigma-1}{\sigma+q}\left(\frac{2(1+q)}{a_{0} C_{\sigma}(\sigma+q)}\right)^{(q+1) /(\sigma-1)} C_{5}^{(\sigma+q) /(\sigma-1)}
\end{aligned}
$$

Рассмотрим теперь член, содержащий производные по $t$. Для этого заметим, что функция $\eta(\tau)$, входящая в определение $\psi(x, t)$, обладает первообразной

$$
\eta_{1}(\tau)= \begin{cases}\frac{1}{2} \tau^{2}, & |\tau| \leqslant 1 \\ \frac{1}{q+1}|\tau|^{q+1}, & |\tau|>1\end{cases}
$$


Действительно,

$$
\begin{aligned}
\left(\frac{1}{q+1}|\tau|^{q+1}\right)_{\tau} & =\frac{1}{2} \frac{2}{q+1}\left(|\tau|^{2((q+1) / 2)}\right)_{\tau}=\frac{1}{2}\left(|\tau|^{2}\right)_{\tau} \cdot\left(|\tau|^{2}\right)^{(q-1) / 2} \\
& =\frac{1}{2} \tau \cdot\left(|\tau|^{2}\right)^{(q-1) / 2}=\eta(\tau) .
\end{aligned}
$$

Поэтому член, содержащий производные по $t$, мы можем записать так:

$$
\begin{aligned}
-\int_{Q} \frac{\partial w_{\varepsilon}(x, t)}{\partial t} \psi(x, t) d x d t & =-\int_{Q} \frac{\partial w_{\varepsilon}(x, t)}{\partial t} \eta\left(w_{\varepsilon}(x, t)\right) \theta_{\varepsilon}^{p}(x) d x d t \\
& =-\int_{Q} \frac{\partial}{\partial t}\left(\eta_{1}\left(w_{\varepsilon}(x, t)\right) \theta_{\varepsilon}^{p}(x)\right) d x d t \\
& =-\left.\int_{\Omega}\left(\eta_{1}\left(w_{\varepsilon}(x, t)\right) \theta_{\varepsilon}^{p}(x)\right)\right|_{0} ^{T} d x \leqslant 0 .
\end{aligned}
$$

Последнее вьполняется, поскольку $\eta_{1}(x, t) \theta_{\varepsilon}^{p}(x)$ положительно и обращается в нуль при $t=0$.

Учитывая равенства $(26),(28)$ и оценки $(29)-(34)$, имеем

$$
\begin{aligned}
\int_{Q} & \left(L w_{\varepsilon}(x, t) \psi(x, t)-\frac{\partial w_{\varepsilon}(x, t)}{\partial t} \psi(x, t)\right) d x d t \\
& \leqslant \int_{Q} L w_{\varepsilon}(x, t) \psi(x, t) d x d t \\
& \leqslant-\frac{1}{2 \lambda} \int_{Q_{1}}\left|\nabla w_{\varepsilon}(x, t)\right|^{2} \theta_{\varepsilon}^{p}(x) d x d t-\frac{q}{2 \lambda} \int_{Q_{2}}\left|\nabla w_{\varepsilon}(x, t)\right|^{2}\left|w_{\varepsilon}(x, t)\right|^{q-1} \theta_{\varepsilon}^{p}(x) d x d t \\
& +C_{4} \int_{Q_{1}}\left|\nabla \theta_{\varepsilon}(x)\right|^{2} \theta_{\varepsilon}^{p-2}(x) d x d t+\frac{a_{0} C_{\sigma}}{2} \int_{Q_{2}}\left|w_{\varepsilon}(x, t)\right|^{\sigma+q} \theta_{\varepsilon}^{p}(x) d x d t \\
& +C_{6} \int_{Q_{2}}\left|\nabla \theta_{\varepsilon}(x)\right|^{p} d x d t
\end{aligned}
$$

Объединяя (26), (27) и (35), получаем

$$
\begin{aligned}
& -\frac{1}{2 \lambda} \int_{Q_{1}}\left|\nabla w_{\varepsilon}(x, t)\right|^{2} \theta_{\varepsilon}^{p}(x) d x d t-\frac{q}{2 \lambda} \int_{Q_{2}}\left|\nabla w_{\varepsilon}(x, t)\right|^{2}\left|w_{\varepsilon}(x, t)\right|^{q-1} \theta_{\varepsilon}^{p}(x) d x d t \\
& \quad+C_{4} \int_{Q_{1}}\left|\nabla \theta_{\varepsilon}(x)\right|^{2} \theta_{\varepsilon}^{p-2}(x) d x d t+\frac{a_{0} C_{\sigma}}{2} \int_{Q_{2}}\left|w_{\varepsilon}(x, t)\right|^{\sigma+q} \theta_{\varepsilon}^{p}(x) d x d t \\
& \quad+C_{6} \int_{Q_{2}}\left|\nabla \theta_{\varepsilon}(x)\right|^{2 \frac{\sigma+q}{\sigma-1}} d x d t \\
& \geqslant a_{0} C_{\sigma} \int_{Q_{1}}\left|w_{\varepsilon}(x, t)\right|^{\sigma+1} \theta_{\varepsilon}^{p}(x) d x d t+a_{0} C_{\sigma} \int_{Q_{2}}\left|w_{\varepsilon}(x, t)\right|^{\sigma+q} \theta_{\varepsilon}^{p}(x) d x d t .
\end{aligned}
$$


После элементарных преобразований получаем

$$
\begin{aligned}
C_{4} \int_{Q_{1}}\left|\nabla \theta_{\varepsilon}(x)\right|^{2} \theta_{\varepsilon}^{p-2}(x) d x d t+C_{6} \int_{Q_{2}}\left|\nabla \theta_{\varepsilon}(x)\right|^{p} d x d t \\
\geqslant a_{0} C_{\sigma} \int_{Q_{1}}\left|w_{\varepsilon}(x, t)\right|^{\sigma+1} \theta_{\varepsilon}^{p}(x) d x d t+\frac{1}{2 \lambda} \int_{Q_{2}}\left|\nabla w_{\varepsilon}(x, t)\right|^{2} \theta_{\varepsilon}^{p}(x) d x d t \\
\quad+\frac{a_{0} C_{\sigma}}{2} \int_{Q_{2}}\left|w_{\varepsilon}(x, t)\right|^{\sigma+q} \theta_{\varepsilon}^{p}(x) d x d t+\frac{q}{2 \lambda} \int_{Q_{2}}\left|\nabla w_{\varepsilon}(x, t)\right|^{2}\left|w_{\varepsilon}(x, t)\right|^{q-1} \theta_{\varepsilon}^{p}(x) d x d t \\
\geqslant C_{7} \int_{Q} \frac{\left|w_{\varepsilon}(x, t)\right|^{\sigma+1}}{1+\left|w_{\varepsilon}(x, t)\right|^{2}} \theta_{\varepsilon}^{p}(x) d x d t+C_{8} \int_{Q} \frac{\left|\nabla w_{\varepsilon}(x, t)\right|^{2}}{1+\left|w_{\varepsilon}(x, t)\right|^{2}} \theta_{\varepsilon}^{p}(x) d x d t \\
\geqslant C_{9} \int_{Q \backslash Q\left(4 R_{\varepsilon}, T\right)} \frac{\left|\nabla w_{\varepsilon}(x, t)\right|^{2}+\left|w_{\varepsilon}(x, t)\right|^{\sigma+1}}{1+\left|w_{\varepsilon}(x, t)\right|^{2}} d x d t
\end{aligned}
$$

где $C_{7}=a_{0} C_{\sigma} / 2, C_{8}=\min \{1 /(2 \lambda), q /(2 \lambda)\}, C_{9}=\min \left\{C_{7}, C_{8}\right\}$.

$\mathrm{K}$ предпоследнему неравенству мы пришли из следующих соображений: если число $0 \leqslant a \leqslant 1$, то вьполнено неравенство $1 \geqslant 1 /\left(1+a^{2}\right)$; если же $a>1$, то вьполнено $a^{q-1}>1 / a^{2}>1 /\left(1+a^{2}\right)$ при $q>-1$.

Теперь докажем, что

$$
\int_{Q_{1}}\left|\nabla \theta_{\varepsilon}(x)\right|^{2} \theta_{\varepsilon}^{p-2}(x) d x d t \rightarrow 0 \quad \text { при } \quad \varepsilon \rightarrow 0
$$

и

$$
\int_{Q_{2}}\left|\nabla \theta_{\varepsilon}(x)\right|^{p} d x d t \rightarrow 0 \quad \text { при } \varepsilon \rightarrow 0 .
$$

Действительно, так как $p>2$, то

$$
\begin{aligned}
\int_{Q_{1}}\left|\nabla \theta_{\varepsilon}(x)\right|^{2} \theta_{\varepsilon}^{p-2}(x) d x d t & \leqslant \int_{Q}\left|\nabla \theta_{\varepsilon}(x)\right|^{2} d x d t \\
& \leqslant C_{10} m R_{\varepsilon}^{n}\left(\frac{1}{R_{\varepsilon}}\right)^{2}=O\left(\varepsilon^{-1+(n-2) \gamma}\right) \rightarrow 0
\end{aligned}
$$

при $\varepsilon \rightarrow 0$, если только $\gamma>1 /(n-2) ;$ где $C_{10}=T C_{\Omega}^{2} \mu_{n} 2^{n}$. Имеем

$$
\begin{aligned}
\int_{Q_{2}}\left|\nabla \theta_{\varepsilon}(x)\right|^{p} d x d t & \leqslant \int_{Q}\left|\nabla \theta_{\varepsilon}(x)\right|^{p} d x d t \leqslant C_{11} m R_{\varepsilon}^{n}\left(\frac{1}{R_{\varepsilon}}\right)^{p} \\
& =C_{11} \varepsilon^{-1} R_{\varepsilon}^{n-p}=O\left(\varepsilon^{-1+(n-p) \gamma}\right) \rightarrow 0
\end{aligned}
$$

при $\varepsilon \rightarrow 0$, так как $\gamma>1 /(n-p)>1 /(n-2)$; здесь $C_{11}=T C_{\Omega}^{2(\sigma+q) /(\sigma-1)} \mu_{n} 2^{n}, \mu_{n}-$ объем единичного $n$-мерного шара.

Из (36)-(38) следует справедливость теоремы 2. 


\section{СПИСОК ЦИТИРОВАННОЙ ЛИТЕРАТУРЫ}

[1] Bensoussan A., Lions J.-L., Papanicolaou G. Asymptotic Analysis for Periodic Structures. Amsterdam: North-Holland, 1978.

[2] ЖКиков В. В., Козлов С. М., Олейник О. А. Усреднение дифференциальных операторов. М.: Физматлит, 1993.

[3] Марченко В.А., Хруслов Е. Я. Краевые задачи в областях с мелкозернистой границей. Киев: Наукова думка, 1974.

[4] Brézis H., Friedman A. Nonlinear parabolic equations involving measures as initial conditions // J. Math. Pures Appl. (9). 1983. V. 62. № 1. P. 73-97.

[5] Brézis H., Peletier L. A., Terman D. A very singular solution of the heat equation with absorption // Arch. Rat. Mech. Anal. 1986. V. 95. № 3. P. 185-209.

[6] Kamin S., Peletier L. A. Singular solutions of the heat equation with absorption // Proc. Amer. Math. Soc. 1985. V. 95. № 2. P. 205-210.

[7] Ильин А.М., Олейник О.А., Калашников А.С. Линейные уравнения второго порядка параболического типа // УМН. 1962. Т. 17. № 3. С. 3-146.

[8] Матевосян О.А., Пикулин С.В. Об усреднении слабонелинейных дивергентных эллиптических операторов в перфорированном кубе // Матем. заметки. 2000. Т. 68. № 3. C. $390-398$.

[9] Матевосян О.А., Пикулин С. В.Об усреднении полулинейных эллиптических операторов в перфорированных областях // Матем. сб. 2002. Т. 193. № 3. С. 101-114.

[10] Кондратьев В. А., Ландис Е. М. О качественных свойствах решений одного нелинейного уравнения второго порядка // Матем. сб. 1988. Т. 135. № 3. С. 346-360.

[11] Чистяков В.В. О свойствах решений полулинейных параболических уравнений второго порядка // Труды семинара И. Г. Петровского. 1991. Т. 15. С. 70-107.

[12] Ладыженская О. А., Солонников В. А., Уральцева Н. Н. Линейные и квазилинейные уравнения параболического типа. М.: Наука, 1967.

[13] Nash J. Continuity of solutions of parabolic and elliptic equations // Amer. J. Math. 1958. V. 80. Р. 931-954; Нэш Дж. О непрерьвности решений параболических и эллиптических уравнений // Математика. 1960. Т. 4. №1. С. 31-52.

[14] Aronson D. G. Bounds for the fundamental solution of a parabolic equation // Bull. Amer. Math. Soc. 1967. V. 73. P. 890-896.

[15] Гильбарг Д., Трудингер Н. Эллиптические дифференциальные уравнения с частными производными второго порядка. М.: Наука, 1989.

Московский государственный университет им. М.В. Ломоносова

Поступило

E-mail : matevossian@cswebmail.com, filimi@yandex.ru

09.06.2003 